\title{
NOTE ON SUMS OF FOUR AND SIX SQUARES
}

\author{
L. CARLITZ
}

1. Bailey [1] showed that Ramanujan's identity

$$
\sum_{m=0}^{\infty} p(5 m+4)=5 \prod_{n=1}^{\infty} \frac{\left(1-x^{5 n}\right)^{5}}{\left(1-x^{n}\right)^{6}}
$$

can be derived from the identity

$$
\begin{aligned}
& \sum_{-\infty}^{\infty}\left\{\frac{x q^{n}}{\left(1-x q^{n}\right)^{2}}-\frac{y q^{n}}{\left(1-y q^{n}\right)^{2}}\right\}=\frac{(x-y)(1-x y)}{(1-x)^{2}(1-y)^{2}} \\
& \cdot \prod_{1}^{\infty} \frac{\left(1-x y q^{n}\right)\left(1-x^{-1} y^{-1} q^{n}\right)\left(1-x y^{-1} q^{n}\right)\left(1-x^{-1} y q^{n}\right)\left(1-q^{n}\right)^{4}}{\left(1-x q^{n}\right)^{2}\left(1-x^{-1} q^{n}\right)^{2}\left(1-y q^{n}\right)^{2}\left(1-y^{-1} q^{n}\right)^{2}}
\end{aligned}
$$

which is equivalent to the familiar formula

$$
\wp(u)-\wp(v)=-\frac{\sigma(u+v) \sigma(u+v)}{\sigma^{2}(u) \sigma^{2}(v)} .
$$

Similarly the formula

$$
\begin{aligned}
1+a^{-1} \frac{(1-a)^{3}}{1+a} \sum_{1}^{\infty} \frac{n^{2} q^{2 n}}{1-q^{2 n}}\left(a^{n}-a^{-n}\right) \\
\quad=\prod_{1}^{\infty} \frac{\left(1-q^{2 n} a^{2}\right)\left(1-q^{2 n} a^{-2}\right)\left(1-q^{2 n}\right)^{6}}{\left(1-q^{2 n} a\right)^{4}\left(1-q^{2 n} a^{-1}\right)^{4}},
\end{aligned}
$$

which is equivalent to

$$
\wp^{\prime}(u)=-\sigma(2 u) / \sigma^{4}(u),
$$

can be used to prove various results involving partition functions. Dobbie [3] recently constructed simple direct proofs of (1) and (2) that require no knowledge of elliptic functions; incidentally (2) can be derived from (1) by dividing by $x-y$ and then letting $y \rightarrow x$.

The writer [2] showed that by means of (2) one can give a very concise proof of the familiar formula for the number of representations of an integer as a sum of eight squares or of eight odd squares. In the present note we obtain the formulas for four and six squares in a similar manner (see for example [6, p. 307]).

Received by the editors February 13, 1956. 
2. We recall the formulas (see for example [5, p. 282])

$$
\begin{aligned}
\theta_{0}(q)=\sum_{-\infty}^{\infty}(-1)^{n} q^{n^{2}} & =\prod^{\infty} \frac{\left(1-q^{n}\right)^{2}}{1-q^{2 n}} \\
\theta_{2}(q)=2 \sum_{1}^{\infty} q^{(2 n-1)^{2 / 4}} & =2 q^{1 / 4} \prod_{1}^{\infty} \frac{\left(1-q^{4 n}\right)^{2}}{1-q^{2 n}} \\
\theta_{3}(q) & =\theta_{0}(-q) .
\end{aligned}
$$

It follows from (3) and (4) that

$$
\theta_{0}(q) \theta_{3}(q)=\theta_{0}^{2}\left(q^{2}\right), \quad \theta_{2}^{2}(q)=2 \theta_{2}\left(q^{2}\right) \theta_{3}\left(q^{2}\right) .
$$

For the case of six squares we shall in addition require

$$
\theta_{3}^{4}(q)=\theta_{0}^{4}(q)+\theta_{2}^{4}(q)
$$

which incidentally is proved in $\$ 3$ below.

We define $r_{k}(n), r_{k}^{\prime}(n)$ by means of

$$
\theta_{3}^{k}(q)=\sum_{n=0}^{\infty} r_{k}(n) q^{n}, \quad \theta_{2}^{k}\left(q^{4}\right)=\sum_{n=1}^{\infty} r_{k}^{\prime}(n) q^{n} .
$$

3. In (1) replace $q$ by $q^{2}$ and then put $y=-x=q$. The left hand side of (1) becomes

$$
\begin{aligned}
& \sum_{n=-\infty}^{\infty}\left\{\frac{q^{2 n+1}}{\left(1-q^{2 n+1}\right)^{2}}+\frac{q^{2 n+1}}{\left(1+q^{2 n+1}\right)^{2}}\right\} \\
& \quad=2 \sum_{n=0}^{\infty}\left\{\frac{q^{2 n+1}}{\left(1-q^{2 n+1}\right)^{2}}+\frac{q^{2 n+1}}{\left(1+q^{2 n+1}\right)^{2}}\right\} .
\end{aligned}
$$

The right hand side of (1) becomes

$$
4 q \prod_{n=1}^{\infty} \frac{\left(1-q^{4 n}\right)^{4}}{\left(1-q^{4 n-2}\right)^{4}}=4 q \prod_{n=1}^{\infty} \frac{\left(1-q^{4 n}\right)^{8}}{\left(1-q^{2 n}\right)^{4}}=\frac{1}{4} \theta_{2}^{4}(q) .
$$

Hence we have the identity

$$
\begin{aligned}
\sum_{n=0}^{\infty} r_{4}^{\prime}(8 n+4) q^{2 n+1}=\theta_{2}^{4}(q) & =8 \sum_{n=0}^{\infty}\left\{\frac{q^{2 n+1}}{\left(1-q^{2 n+1}\right)^{2}}+\frac{q^{2 n+1}}{\left(1-q^{2 n+1}\right)^{2}}\right\} \\
& =16 \sum_{n=0}^{\infty} \sum_{r=0}^{\infty}(2 r+1) q^{(2 n+1)(2 r+1)}
\end{aligned}
$$

which is equivalent to the known results on sums of four odd squares.

In (1) let us now put $x=i$ and $y=-i$. The left hand side of (1) becomes 


$$
\begin{aligned}
\sum_{n=-\infty}^{\infty}\left\{\frac{i q^{n}}{\left(1-i q^{n}\right)^{2}}\right. & \left.+\frac{q^{n}}{\left(1+q^{n}\right)^{2}}\right\} \\
& =-\frac{1}{4}+\sum_{n=1}^{\infty}\left\{\frac{i q^{n}}{\left(1-i q^{n}\right)^{2}}-\frac{i q^{n}}{\left(1+i q^{n}\right)^{2}}+\frac{2 q^{n}}{\left(1+q^{n}\right)^{2}}\right\} \\
& =-\frac{1}{4}+\sum_{n=1}^{\infty}\left\{-\frac{4 q^{2 n}}{\left(1+q^{2 n}\right)^{2}}+\frac{2 q^{n}}{\left(1+q^{n}\right)^{2}}\right\} \\
& =-\frac{1}{4}-2 \sum_{n=1}^{\infty}(-1)^{n} \frac{q^{n}}{\left(1+q^{n}\right)^{2}} .
\end{aligned}
$$

The right hand side of (1) becomes

$$
\begin{aligned}
&-\frac{1}{4} \prod_{n=1}^{\infty} \frac{\left(1+i q^{n}\right)^{2}\left(1-i q^{n}\right)^{2}\left(1-q^{n}\right)^{4}}{\left(1-i q^{n}\right)^{2}\left(1+i q^{n}\right)^{2}\left(1+q^{n}\right)^{4}} \\
& \quad=-\frac{1}{4} \prod_{n=1}^{\infty} \frac{\left(1-q^{n}\right)^{8}}{\left(1-q^{2 n}\right)^{4}}=-\frac{1}{4} \theta_{0}^{4}(q) .
\end{aligned}
$$

Hence we have the identities

$$
\begin{aligned}
\theta_{0}^{4}(q) & =1+8 \sum_{n=1}^{\infty} \frac{(-1)^{n} q^{n}}{\left(1+q^{n}\right)^{2}}=1+8 \sum_{n=1}^{\infty} \sum_{r=1}^{\infty}(-1)^{n+r+1} r q^{n r} \\
\sum_{n=0}^{\infty} r_{4}(n) q^{n} & =\theta_{3}^{4}(q)=\theta_{0}^{(4)}(-q)=1+8 \sum_{n=1}^{\infty} \frac{q^{n}}{\left(1+(-q)^{n}\right)^{2}} \\
& =1+8 \sum_{n=1}^{\infty} \sum_{r=1}^{\infty}(-1)^{(n-1)(r-1)} r q^{n r}
\end{aligned}
$$

the latter of which is equivalent to the known results on sums of four squares.

From (9), (10) and (11) we see that

$$
\theta_{3}^{4}(q)=\theta_{0}^{4}(q)+\theta_{2}^{4}(q),
$$

which is (7) above. The writer is indebted to the referee for this observation.

4. Turning next to (2), we take $a=i$. This yields

$$
1-4 \sum_{m=1}^{\infty}\left(\frac{-4}{m}\right) \frac{m^{2} q^{2 m}}{1-q^{2 m}}=\prod_{1}^{\infty} \frac{\left(1-q^{2 n}\right)^{4}\left(1-q^{4 n}\right)^{6}}{\left(1-q^{8 n}\right)^{4}}
$$

where $(-4 / m)$ is the Jacobi symbol. The right member is equal to

$$
\prod_{1}^{\infty} \frac{\left(1-q^{2 n}\right)^{4}\left(1-q^{4 n}\right)^{8}}{\left(1-q^{4 n}\right)^{2}\left(1-q^{8 n}\right)^{4}}=\theta_{0}^{2}\left(q^{2}\right) \theta_{0}^{4}\left(q^{4}\right)=\theta_{0}^{4}\left(q^{2}\right) \theta_{3}^{2}\left(q^{2}\right),
$$


where we have used (3) and (6). We have therefore

$$
1-4 \sum_{1}^{\infty}\left(\frac{-4}{m}\right) \frac{m^{2} q^{2 m}}{1-q^{2 m}}=\theta_{0}^{4}\left(q^{2}\right) \theta_{3}^{2}\left(q^{2}\right) \text {. }
$$

Now take $a=q i$ in (2). We find that the right member becomes

$$
2 \frac{(1-q i)^{4}}{1+q^{2}} \prod_{1}^{\infty} \frac{\left(1-q^{2 n}\right)^{8}\left(1-q^{8 n}\right)^{4}}{\left(1-q^{4 n}\right)^{6}}=\frac{(1-q i)^{3}}{1+q i} \frac{\theta_{0}^{4}\left(q^{2}\right) \theta_{2}^{2}\left(q^{2}\right)}{2 q}
$$

the left member is equal to

$$
\begin{aligned}
1+\frac{1}{q} & \frac{(1-q i)^{3}}{1+q i} \sum_{1}^{\infty} \frac{n^{2} q^{2 n}}{1-q^{2 n}} i^{n-1}\left(q^{n}-(-1)^{n} q^{-n}\right) \\
= & \frac{(1-q i)^{3}}{q(1+q i)}\left\{\frac{q(1+q i)}{(1-q i)^{3}}+\sum_{1}^{\infty} \frac{n^{2} q^{2 n}}{1-q^{2 n}} i^{n-1}\left(q^{n}-(-1)^{n} q^{-n}\right)\right\} \\
= & 2 \frac{(1-q i)^{3}}{q(1+q i)} \sum_{1}^{\infty} \sum_{1}^{\infty}(-1)^{n-1}(2 n-1)^{2} q^{(2 r-1)(2 n-1)},
\end{aligned}
$$

on expanding and combining. Thus we get

$$
4 \sum_{1}^{\infty} \sum_{1}^{\infty}(-1)^{n-1}(2 n-1)^{2} q^{(2 r-1)(2 n-1)}=\theta_{0}^{4}\left(q^{2}\right) \theta_{2}^{2}\left(q^{2}\right) .
$$

If we divide by $q$, replace $q^{2}$ by $-q^{2}$, we find that (13) becomes

$$
4 \sum_{1}^{\infty} \sum_{1}^{\infty}(-1)^{r-1}(2 n-1)^{2} q^{(2 r-1)(2 n-1)}=\theta_{3}^{4}\left(q^{2}\right) \theta_{2}^{2}\left(q^{2}\right) .
$$

Again, if we take $a=q^{1 / 2}$ in (2) and then replace $q$ by $q^{4}$, we get without much difficulty

$$
\begin{gathered}
1+q^{-2} \frac{\left(1-q^{2}\right)^{2}}{1+q^{2}} \sum_{1}^{\infty} \frac{n^{2} q^{6 n}}{1+q^{4 n}}=\frac{\left(1-q^{2}\right)^{3}}{1+q^{2}} \prod_{1}^{\infty} \frac{\left(1-q^{8 n}\right)^{4}\left(1-q^{4 n}\right)^{8}}{\left(1-q^{4 n}\right)^{2}\left(1-q^{2 n}\right)^{4}} \\
64 q^{2} \frac{1+q^{2}}{\left(1-q^{2}\right)^{3}}+64 \sum_{1}^{\infty} \frac{n^{2} q^{6 n}}{1+q^{4 n}}=\theta_{2}^{2}\left(q^{2}\right) \theta_{2}^{4}(q)
\end{gathered}
$$

hence by the second of (6)

$$
16 q^{2} \frac{1+q^{2}}{\left(1-q^{2}\right)^{3}}+16 \sum_{1}^{\infty} \frac{n^{2} q^{6 n}}{1+q^{4 n}}=\theta_{3}^{2}\left(q^{2}\right) \theta_{2}^{4}\left(q^{2}\right) .
$$

If we subtract (13) from (14) and use (7), it is evident that 


$$
\theta_{2}^{6}\left(q^{2}\right)=4 \sum_{1}^{\infty} \sum_{1}^{\infty}\left\{(-1)^{r-1}-(-1)^{s-1}\right\}(2 s-1)^{2} q^{(2 r-1)(2 s-1)}
$$

Define

$$
E_{2}(n)=\sum_{d \mid n}\left(\frac{-4}{d}\right) d^{2}, \quad E_{2}^{\prime}(n)=\sum_{d \delta=n}\left(\frac{-4}{d}\right) \delta^{2} ;
$$

then the right member of (16) becomes

$$
4 \sum_{m \text { odd }} q^{m}\left\{E_{2}^{\prime}(m)-E_{2}(m)\right\} .
$$

This evidently implies

$$
r_{6}^{\prime}(2 m)=4\left\{E_{2}^{\prime}(m)-E_{2}(m)\right\} \quad \text { (m odd). }
$$

On the other hand, addition of (12) and (15) gives after some simplification

$$
\theta_{6}^{6}\left(q^{2}\right)=1+16 \sum_{1}^{\infty} \sum_{1}^{\infty}\left(\frac{-4}{r}\right) n^{2} q^{2 n r}-4 \sum_{1}^{\infty} \sum_{1}^{\infty}\left(\begin{array}{c}
-4 \\
n
\end{array}\right) n^{2} q^{2 n r},
$$

which implies

$$
r_{6}(n)=16 E_{2}^{\prime}(n)-4 E_{2}(n) .
$$

The formulas (17) and (18) are the well-known results of Jacobi on six squares; the notation is that of Glaisher [4].

We remark that (14) and (15) imply results on the number of representations in the forms

$$
4\left(x_{1}^{2}+\dot{x}_{2}^{2}+x_{3}^{2}+x_{4}^{2}\right)+u_{1}^{2}+\underset{u_{2}^{2},}{2} \quad 4\left(x_{1}^{2}+x_{2}^{2}\right)+u_{1}^{2}+u_{2}^{2}+u_{3}^{2}+u_{4}^{2},
$$

where the $u_{i}$ are odd, $x_{i}$ arbitrary.

\section{REFERENCES}

1. W. N. Bailey, A further note on two of Ramanujan's identities, Quart. J. Math. Oxford Ser. (2) vol. 3 (1952) pp. 158-160.

2. L. Carlitz, Some partition formulas related to sums of squares, Nieuw Archief voor Wiskunde (3) vol. 3 (1955) pp. 129-133.

3. J. M. Dobbie, A simple proof of some partition formulae of Ramanujan, Quart. J. Math. Oxford Ser (2) vol. 6 (1955) pp. 193-196.

4. J. W. L. Glaisher, On the number of representations of a number as a sum of $2 r$ squares, where $2 r$ does not exceed eighteen, Proc. London Math. Soc. vol. 5 (1907) pp. $479-490$.

5. G. H. Hardy and E. M. Wright, Introduction to the theory of numbers, Oxford, 1938.

6. H. J. S. Smith, Report on the theory of numbers, Collected Mathematical Papers, vol. 1, Oxford, 1894.

DUKE UNIVERSITY 